Supporting Information

\title{
Sulfonated polycatechol immobilized in a
}

conductive polymer for enhanced energy storage

Daniil A. Lukyanov*, Anatoliy A. Vereshchagin, Anastasiya V. Soloviova, Olga V.

Grigorova, Petr S. Vlasov, Oleg V. Levin*.

Institute of Chemistry, St. Petersburg state University, 199034 St. Petersburg, Russia.

Corresponding Author

*E-mail: o.levin@spbu.ru

*E-mail: Ida93@yandex.ru 
Table S1. Energy storage performance of the representative materials consisted of CP doped with anionic polycatechols.

\begin{tabular}{|c|c|c|c|c|c|}
\hline Material & Electrolyte & $\begin{array}{l}\text { Specific } \\
\text { capacity }\end{array}$ & $\begin{array}{c}\text { Capacity } \\
\text { assessment } \\
\text { conditions }\end{array}$ & Stability & Ref. \\
\hline PEDOT:SL ${ }^{a}$ & $0.1 \mathrm{M} \mathrm{HClO}_{4} / \mathrm{H}_{2} \mathrm{O}$ & $34.1 \mathrm{mAh} \mathrm{g}^{-1}$ & GCD $1 \mathrm{~A} \mathrm{~g}^{-1}$ & $\begin{array}{l}85 \% \text { retention after } \\
1000 \text { cycles at } 8 \mathrm{~A} \mathrm{~g}^{-1}\end{array}$ & [1] \\
\hline PEDOT:SL & $0.1 \mathrm{M} \mathrm{HClO}_{4} / \mathrm{H}_{2} \mathrm{O}$ & $44.6 \mathrm{mAh} \mathrm{g}^{-1}$ & GCD $1 \mathrm{~A} \mathrm{~g}^{-1}$ & - & [2] \\
\hline PPy $^{b)}: S L$ & $0.1 \mathrm{M} \mathrm{HClO}_{4} / \mathrm{H}_{2} \mathrm{O}$ & 73 mAh g $^{-1}$ & $0.83 \mathrm{~A} \mathrm{~g}^{-1}$ & $\begin{array}{l}98 \% \text { retention after } \\
200 \text { cycles at } 1 \mathrm{~A} \mathrm{~g}^{-1}\end{array}$ & [3] \\
\hline $\begin{array}{l}\text { PEDOT:P(4VC- } \\
36)^{d)}\end{array}$ & $0.2 \mathrm{M} \mathrm{H}_{2} \mathrm{SO}_{4} / \mathrm{H}_{2} \mathrm{O}$ & $52 \mathrm{mAh} \mathrm{g}^{-1}$ & $\begin{array}{c}\mathrm{CV} \text { at a scan } \\
\text { rate of } \\
10 \mathrm{mV} \mathrm{s}^{-1}\end{array}$ & $\begin{array}{c}83 \% \text { retention after } \\
200 \mathrm{CV} \text { cycles at } \\
10 \mathrm{mV} \mathrm{s}^{-1}\end{array}$ & [4] \\
\hline PEDOT-Q ${ }^{\mathrm{e}}$ & $\begin{array}{l}\text { Aqueous } \mathrm{NaNO}_{3} \\
\text { buffer }(\mathrm{pH} 0)\end{array}$ & $101 \mathrm{mAh} \mathrm{g}^{-1}$ & $\begin{array}{l}\mathrm{CV} \text { at a scan } \\
\text { rate of } \\
25 \mathrm{mV} \mathrm{s}^{-1} \\
\end{array}$ & - & [5] \\
\hline PEDOT:SPVQ & $0.1 \mathrm{M} \mathrm{HClO}_{4} / \mathrm{H}_{2} \mathrm{O}$ & $49 \mathrm{mAh} \mathrm{g}^{-1}$ & $\begin{array}{c}\mathrm{CV} \text { at a scan } \\
\text { rate of } \\
5 \mathrm{mV} \mathrm{s}^{-1} \\
\mathrm{GCD} \text { at } \\
6 \mathrm{mAh} \mathrm{g}^{-1}\end{array}$ & $\begin{array}{c}70 \% \text { retention after } \\
100 \text { cycles, } 35 \% \text { after } \\
1500 \text { cycles of CV at } \\
50 \mathrm{mV} \mathrm{s}^{-1}\end{array}$ & $\begin{array}{l}\text { This } \\
\text { work }\end{array}$ \\
\hline
\end{tabular}

a) SL - sulfonated lignin b) PPy - polypyrrole c) PANI - polyaniline d) P(4VC-36) - copolymer of 4-vinylcatechol and styrenesulfonic acid e) PEDOT-Q - quinone covalently linked to the PEDOT chain f) SPVQ - sulfonated poly(3,4dihydroxystyrene)

1. Ajjan, F. N.; Casado, N.; Rębiś, T.; Elfwing, A.; Solin, N.; Mecerreyes, D.; Inganäs, O., High performance PEDOT/lignin biopolymer composites for electrochemical supercapacitors. Journal of Materials Chemistry A 2016, 4 (5), 1838-1847.

2. $\quad$ Che, C.; Vagin, M.; Ail, U.; Gueskine, V.; Phopase, J.; Brooke, R.; Gabrielsson, R.; Jonsson, M. P.; Mak, W. C.; Berggren, M.; Crispin, X., Twinning Lignosulfonate with a Conducting Polymer via CounterIon Exchange for Large-Scale Electrical Storage. Advanced Sustainable Systems 2019, 3 (9).

3. Nagaraju, D. H.; Rebis, T.; Gabrielsson, R.; Elfwing, A.; Milczarek, G.; Inganäs, O., Charge storage capacity of renewable biopolymer/conjugated polymer interpenetrating networks enhanced by electroactive dopants. Advanced Energy Materials 2014, 4 (1).

4. $\quad$ Chhin, D.; Padilla-Sampson, L.; Malenfant, J.; Rigaut, V.; Nazemi, A.; Schougaard, S. B., Conducting Polymers Doped with Bifunctional Copolymers for Improved Organic Batteries. ACS Applied Energy Materials 2019, 2 (11), 7781-7790.

5. Sterby, M.; Emanuelsson, R.; Huang, X.; Gogoll, A.; Strømme, M.; Sjödin, M., Characterization of PEDOT-Quinone Conducting Redox Polymers for Water Based Secondary Batteries. Electrochim. Acta 2017, 235, 356-364. 


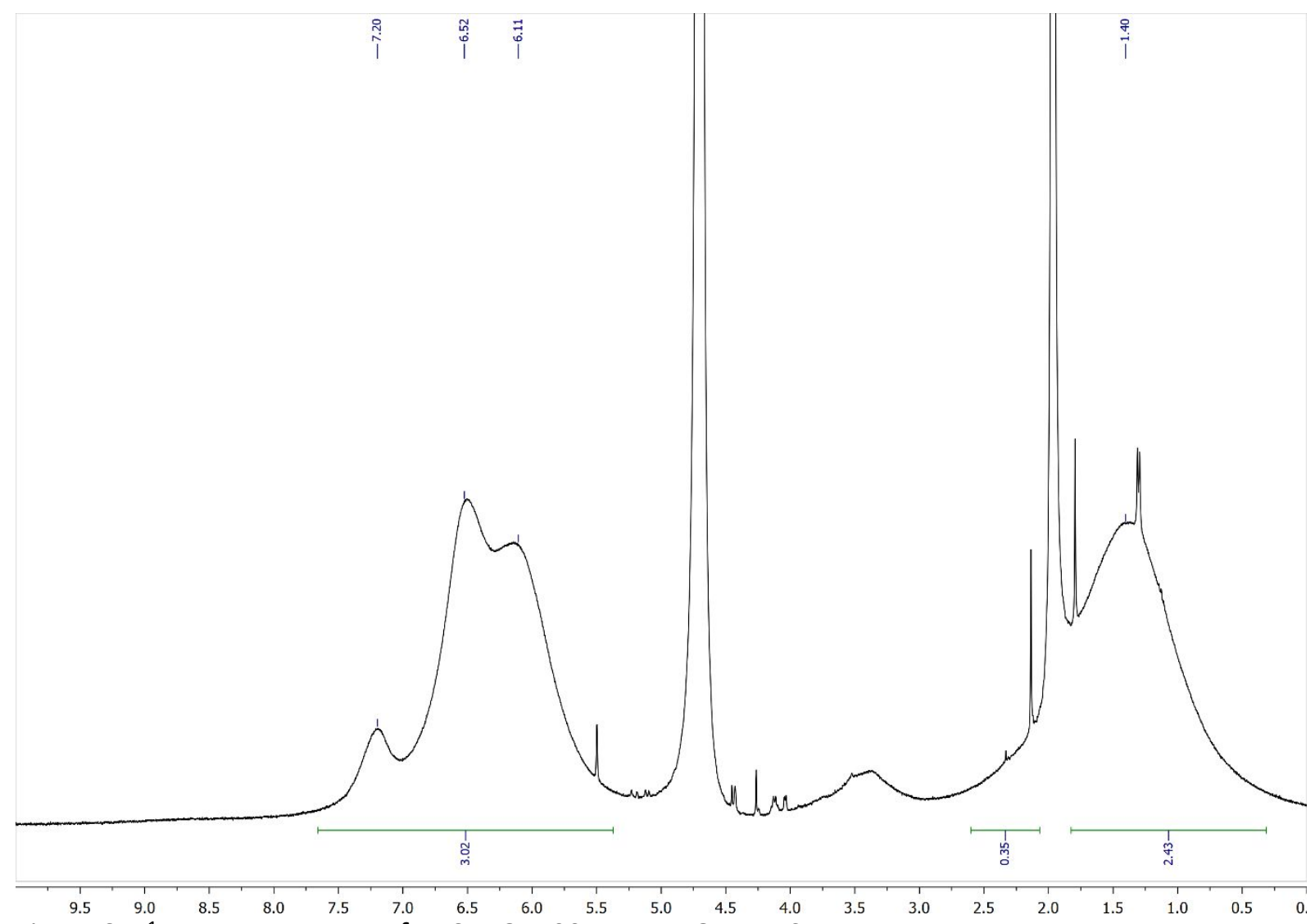

Figure S1. ${ }^{1} \mathrm{H}$ NMR spectrum of NaSPVQ, $400 \mathrm{MHz}, \mathrm{D}_{2} \mathrm{O}+\mathrm{MeCN}$

ФSHIMADZU

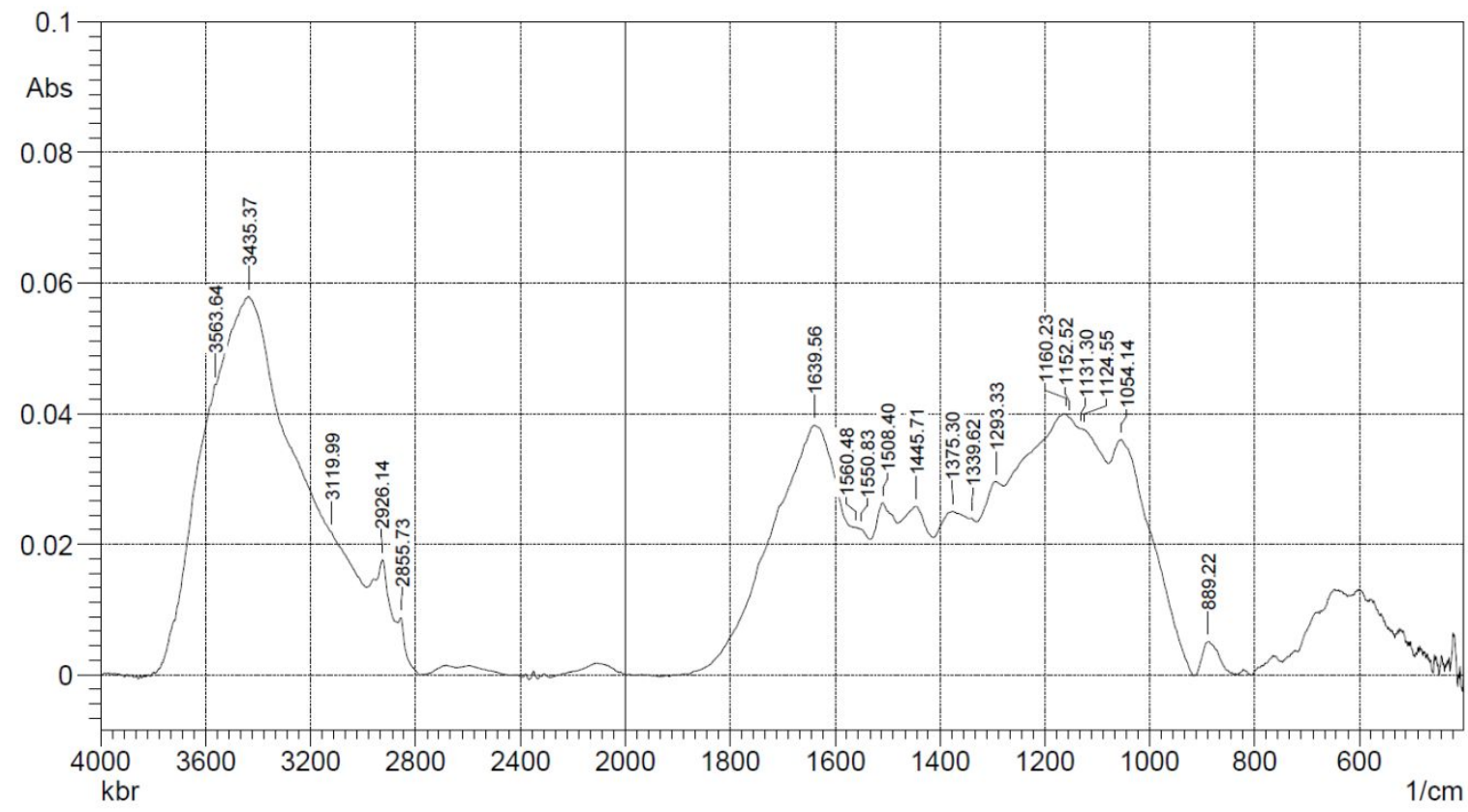

Figure S2. FTIR spectrum of NaSPVQ. 


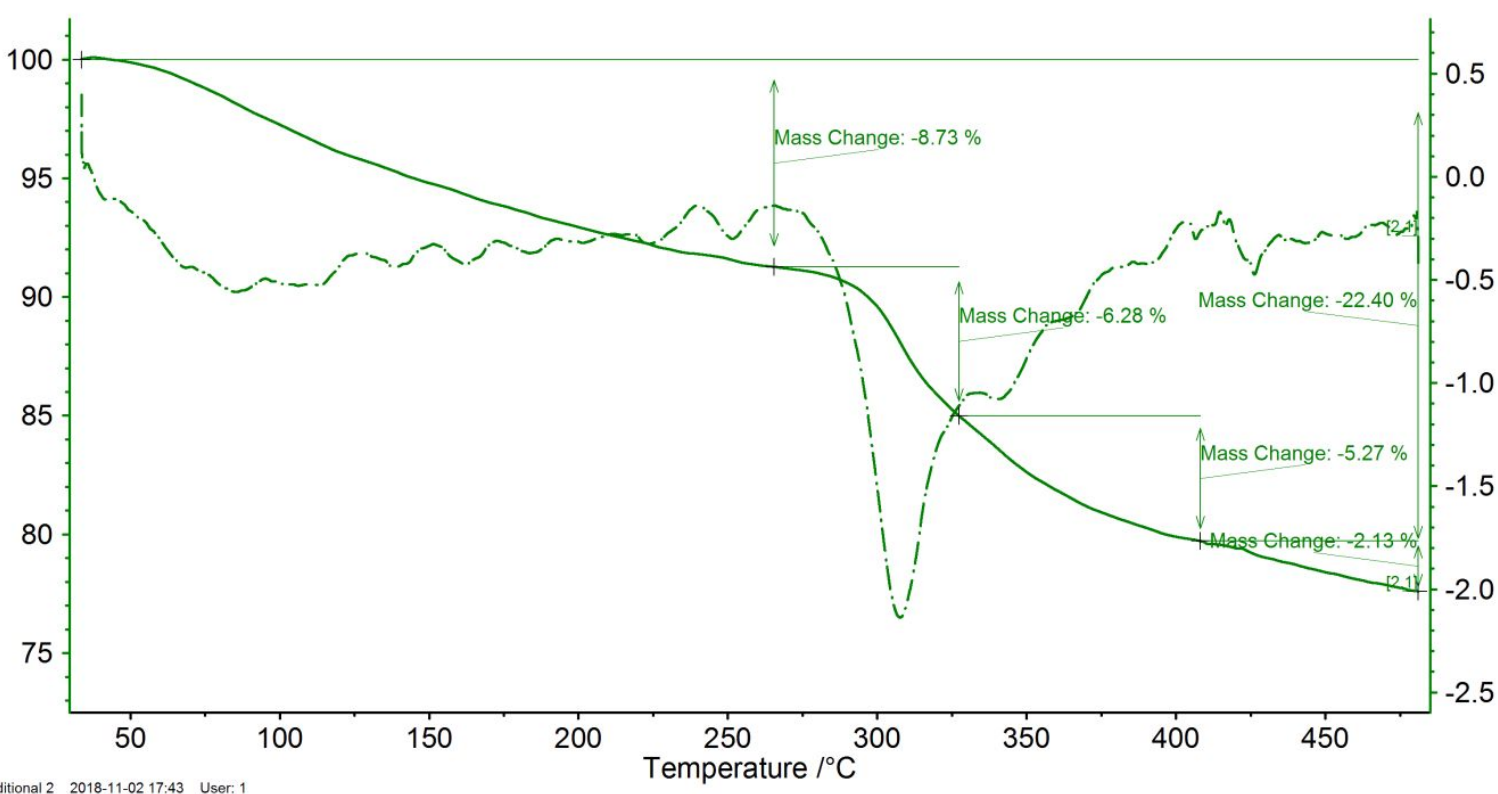

Additional 2 2018-11-02 17:43 User: 1

Instrument : NETZSCH TG 209F1 Libra TGA209... File : C:INE ...1114-10250-2027-04-03-LDA_SPQ-40-510C-10Kmin-Al-Ar-50-0-Ar-50.ngb-dt9 Remark : 114-10250-2027-04-03-LDA_SPQ-40-510C-10KminProject: 114-9135-1998 Material : LDA_SPQ

Prentity: $\quad 114-10250-2027-04-03$

Laboratory : RC Thermo

Operator: Chislov $\quad$ Sample car./TC: TG 209F

Correction file : $114-10250-2027-04-02-e m p t y-40-510 \mathrm{C}-10 \mathrm{Kmin}-\mathrm{Al}-\mathrm{Ar}-50-0-\mathrm{Ar}-50 . \mathrm{ngb}-\mathrm{bt}$

Temp. calib. file : 2015-02-10-Temp-calib^10K-min-Al2O3-50-50-Air.ngb-tt9

Range :

$30^{\circ} \mathrm{C} / 10.0(\mathrm{~K} / \mathrm{min}) / 520^{\circ} \mathrm{C}$ Modeltype of meas. : TG/sample with correction

$\begin{array}{ll}\text { Mode/type of meas. : } & \text { TG/ } \\ \text { Segments : } & 1 / 4\end{array}$

Crucible :

Atmosphere:

Corrm.

Al

Sample mass : $1.13 \mathrm{mg}$

OxVac

Created with NETZSCH Proteus soffware

Figure S3. TGA/DSC curves of NaSPVQ.

TG $/ \%$

Flow $/(\mathrm{ml} / \mathrm{min})$

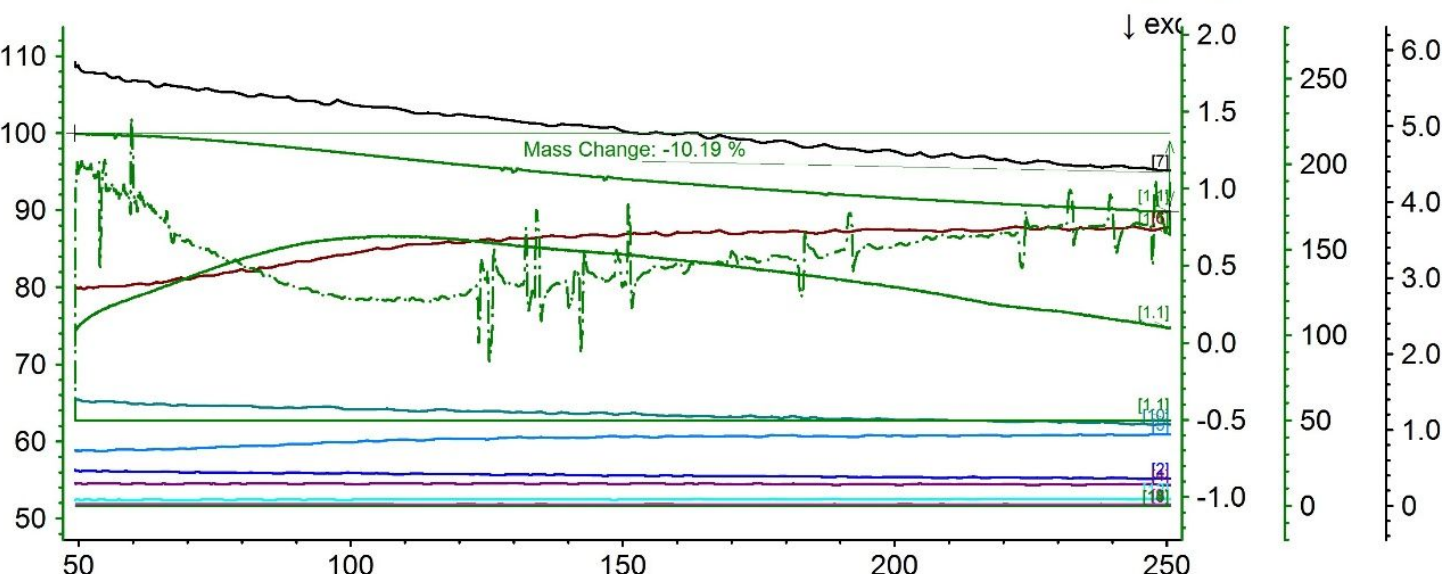

Main 2018-11-09 16:47 User: a

[\#] Instrume... File
[1.1] STA 44... 114-10250-2034-01-03-LDA-SPQ-40-250C-10K-Al-Ar....

Temperature $/{ }^{\circ} \mathrm{C}$

[2] QMS 403 114-10250-2034-01-03-Ida-spq-40_m14.00_s1.imp

114-10250-2034-01-03-1da-spq-40_m15.00_s1. Imp

4] QMS 403 114-10250-2034-01-03-da-spq-40_m16.00_s1.imp

1. QMS 403 114-10250-2034-01-03-da-spq-40_m17.00_s1. Imp

114-10250-2034-01-03-lda-spq-40_mis.00_si.mp

1) QMS 403 114-10250-2034-01-03-lda-spq-40_m28.00_s1.imp

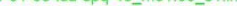

114-10250-2034-01-03-1da-spq-40-m30.00-s1.imp

[1. QMS 403 114-10250-2034-01-03-lda-spq-40_m32.00 s1.imp

14] QMS 403 114-10250

[15] QMS 403 114-10250-2034-01-03-Ida-spq-40_m64.00_s1.im

2018-11... $114-1025$

2018-11... 114-10250-2034-01-03-dda-s... Mass $15 . . .11 .50^{\circ} \mathrm{C} / 9.1(\mathrm{~K} / \mathrm{min}) / 25$.

2018-11.. 114-10250-2034-01-03-daa-s... Mass $16 . .$.

2018-11... 114-10250-2034-01-03-da-s... Mass $17 . .$.

2018-11... 114-10250-2034-01-03-da-s... Mass $18 . .$.

2018-11... 114-10250-2034-01-03-lda-s... Mass $23 .$.

2018-11... 114-10250-2034-01-03-dad-s... Mas. Mas

2018-11... 114-10250-2034-01-03-dda-s... Mass $30 .$.

2018-11... 114-10250-2034-01-03-dda-s... Mass 32.

2018-11... 114-10250-2034-01-03--da-5... Mass 44 .

2018-11... 114-10250-2034-01-03-dda-s... Mass 46.

\begin{tabular}{l|ll}
$2018-11 \ldots$ & $114-10250-2034-01-03-$ dda-s... & Mass 48. \\
$2018-11 \ldots$ & $114-10250-2034-01-03-$ dda-s... & Mass 64.
\end{tabular}

$5007(\mathrm{~K} / \mathrm{min}) 25$

$50^{\circ} \mathrm{C} / 9.1(\mathrm{~K} / \mathrm{min}) / 25$

Figure S4. Thermal MS analysis of NaSPVQ. 


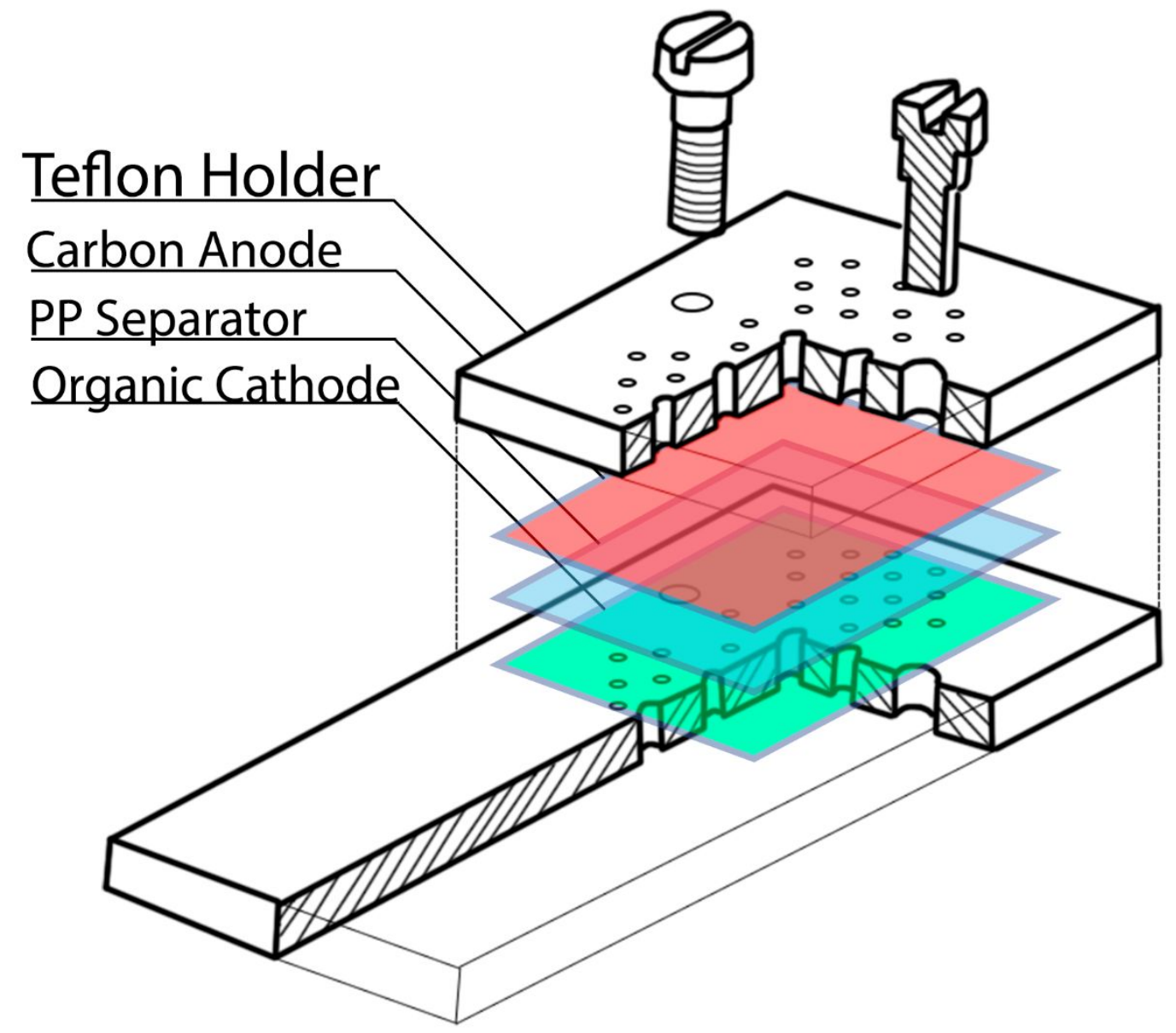

Figure S5. Teflon prototype cell for carbon fiber electrodes.
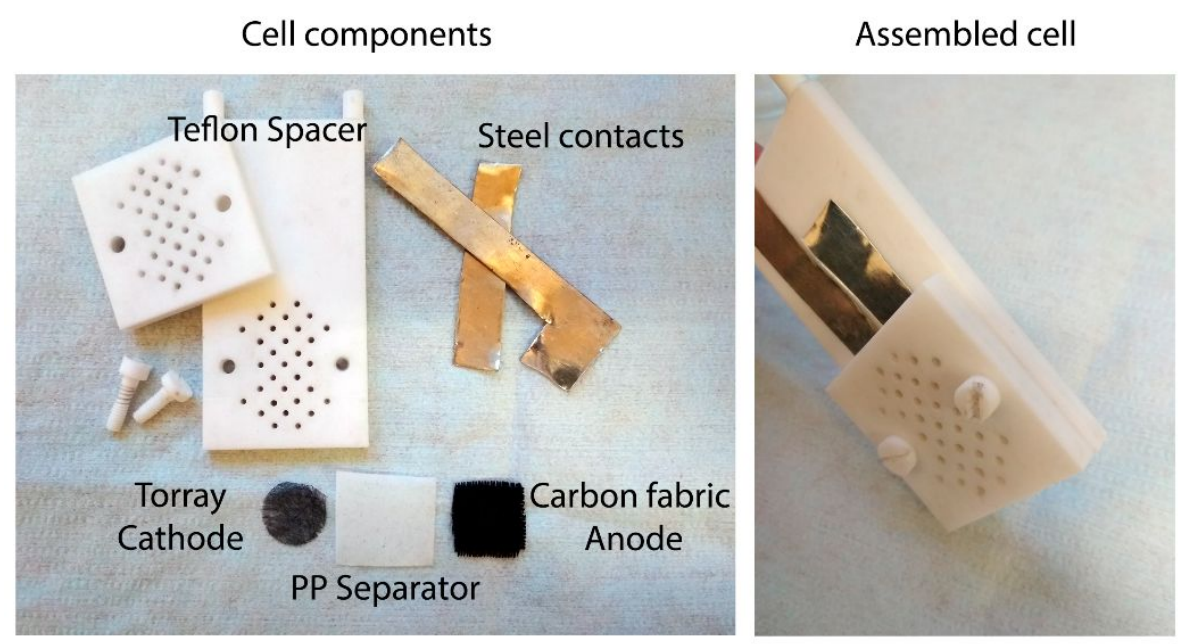

Working cell

Figure S6. Teflon prototype cell for carbon fiber electrodes. 


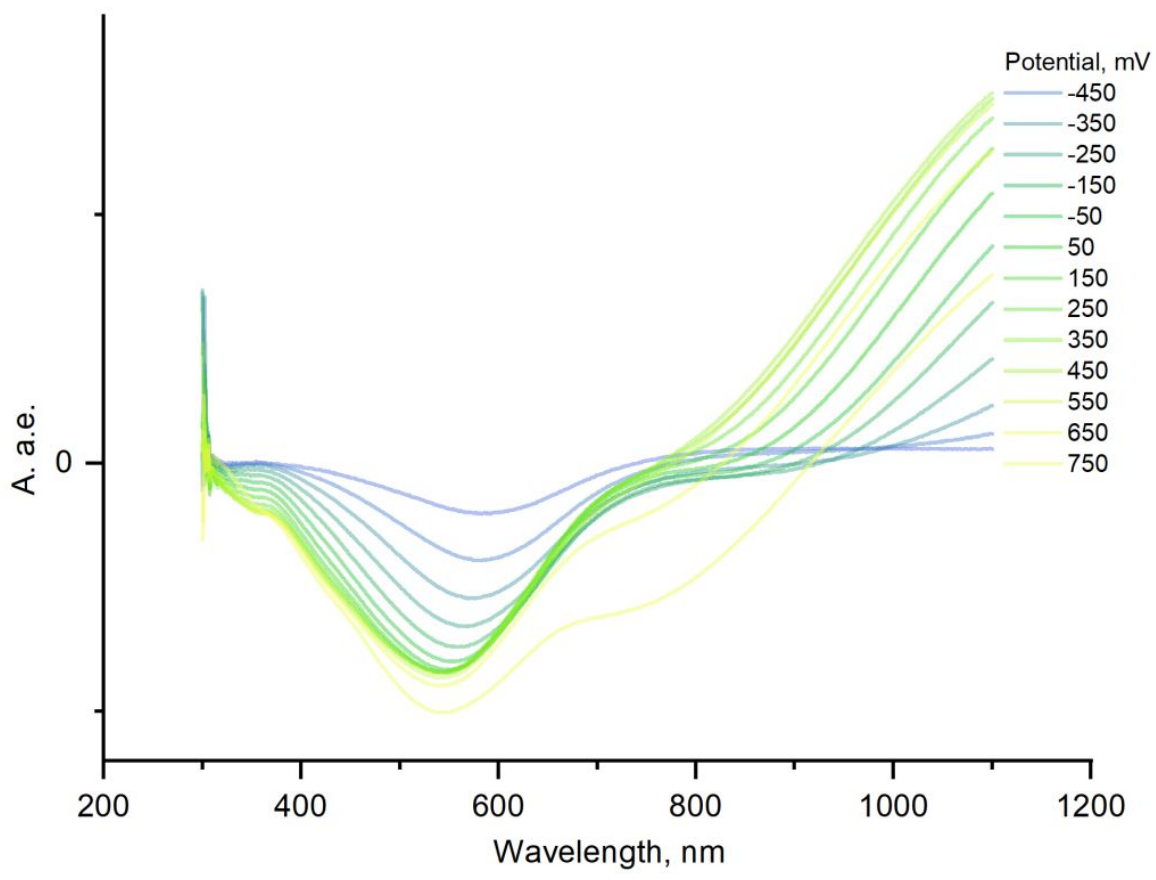

Figure S7. Difference representation of UV-Vis spectroelectrochemistry of PEDOT:PSS.

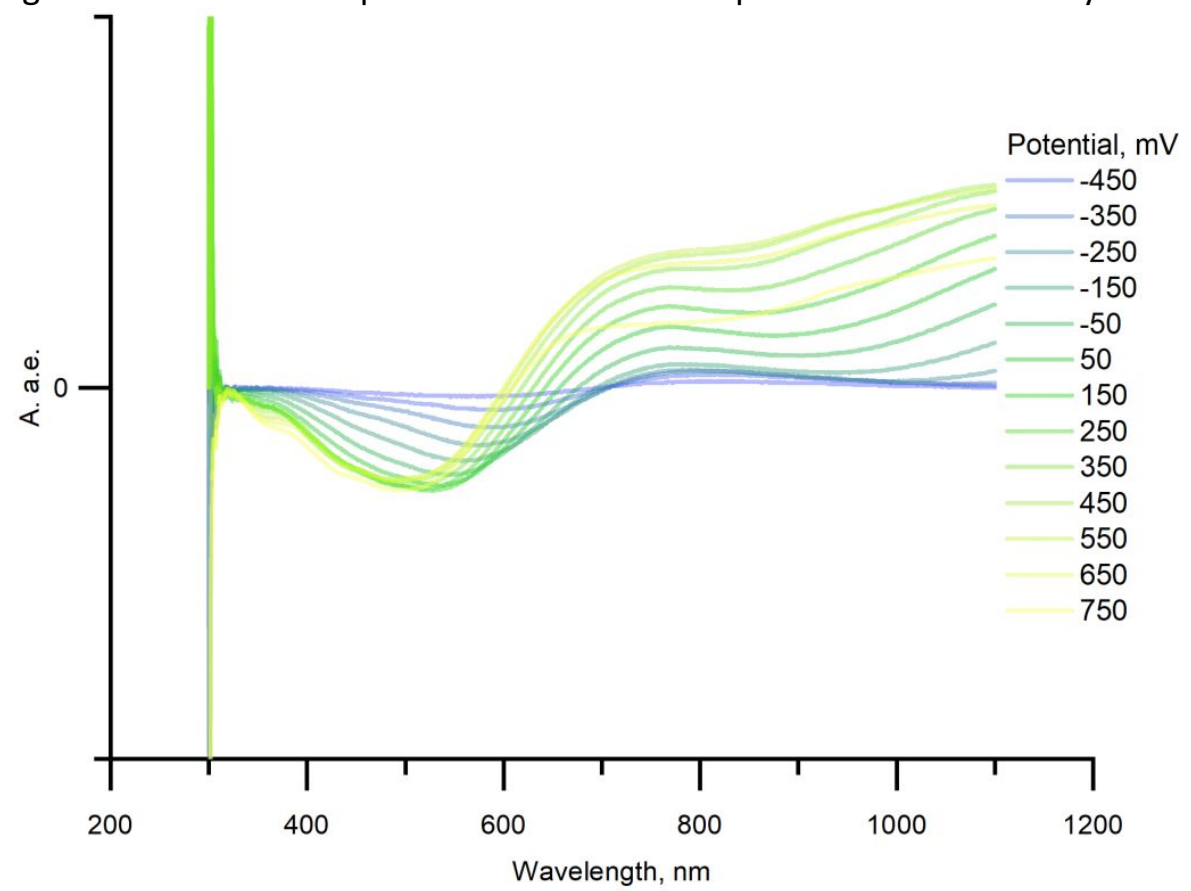

Figure S8. Difference representation of UV-Vis spectroelectrochemistry of PEDOT:SPVQ. 


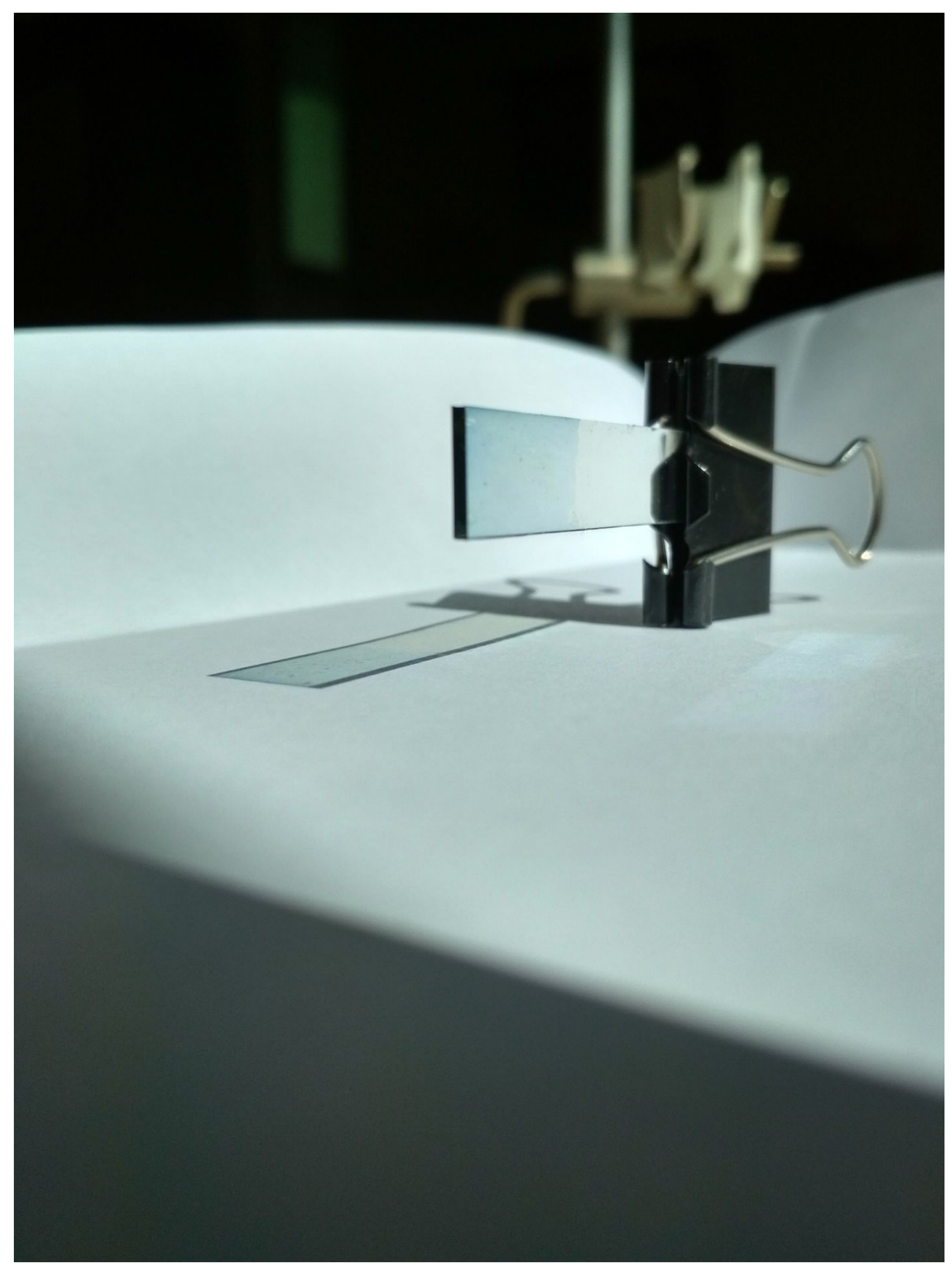

Figure S9. PEDOT:SPVQ film deposited on ITO. 